\title{
EFFECT OF MAGNETIC FIELD ON THERMAL INSTABILITY OF OLDROYDIAN VISCOELASTIC ROTATING FLUID IN POROUS MEDIUM
}

\author{
R.C. THAKUR \\ Department of Mathematics \\ Government P.G. College Dhaliara- 177103 \\ Himachal Pradesh, INDIA \\ E-mail: rameshnahan@yahoo.com \\ G.C. RANA* \\ Department of Mathematics \\ NSCBM Govt. P. G. College, Hamirpur-177 005 \\ Himachal Pradesh, INDIA \\ E-mail: drgcrana15@gmail.com
}

\begin{abstract}
In this paper, we investigate the effect of a vertical magnetic field on thermal instability of an Oldroydian visco-elastic rotating fluid in a porous medium. By applying the normal mode analysis method, the dispersion relation governing the effects of rotation, magnetic field and medium permeability is derived and solved analytically and numerically. For the case of stationary convection, the Oldroydian viscoelastic fluid behaves like an ordinary Newtonian fluid and it is observed that rotation has a stabilizing effect while the magnetic field and medium permeability have a stabilizing/destabilizing effect under certain conditions on thermal instability of the Oldroydian viscoelastic fluid in a porous medium. The oscillatory modes are introduced due to the presence of rotation, the magnetic field and gravity field. It is also observed that the 'principle of exchange of stability' is invalid in the presence of rotation and the magnetic field.
\end{abstract}

Key words: magnetic field, Oldroydian visco-elastic fluid, porous medium, rotation, thermal instability.

Mathematics Subject Classification: 76A10, 76D50, 76E25, 76S05.

\section{Introduction}

In recent years, they has been considerable interest in the study of thermal instability in a porous medium, because it has various applications in geophysics, food processing, soil sciences, ground water hydrology and astrophysics. A detailed account of thermal instability of Newtonian fluids in the presence of rotation and the magnetic field has been given in detail by Chanderasekher (1981). The problem of convective instability of a viscoelastic fluid heated from below was first studied by Green (1968). Vest and Arpaci (1969) investigated the problem of overstability of a viscoelastic fluid heated from below.

Bhatia and Steiner (1972) studied the problem of thermal instability of an Oldroydian visco-elastic fluid in hydromagnetics and found that the magnetic field has a stabilizing influence on the visco-elastic fluid just as in the case of a Newtonian fluid. There are many common materials such as paints, polymers, plastics, and magma, saturated soils and Earth's lithosphere which behave as viscoelastic fluids. In the condition of shear flow, viscoelastic fluids develop transverse normal stresses that cause many phenomena as shear thinning or thickening.

A good account of convection problems in a porous medium is given by Vafai and Hadim (2000), Ingham and Pop (1981) and Nield and Bejan (2006). The Rayleigh instability of a thermal boundary layer in the flow in a porous medium is studied by Wooding (1960). Sharma (1975) studied thermal instability of a

\footnotetext{
* To whom correspondence should be addressed
} 
viscoelastic fluid in hydromagnetics. Sharma (1975) also studied the effect of rotation on thermal instability of a viscoelastic fluid and found that rotation has a stabilizing effect. Rana and Kumar (2000) studied thermal instability of a Rivlin-Ericksen rotating fluid permeated with suspended particles in a porous medium whereas the effect of rotation on thermal instability of compressible Walters' (Model B') fluid was studied by Rana and Kango (2011).

Our main aim in the present paper is to study the effect of the magnetic field on thermal instability of an Oldroydian viscoelastic rotating fluid in a porous medium.

\section{Mathematical model and perturbation equations}

Consider an infinite horizontal layer of an Oldroydian viscoelastic fluid of thickness ' $d$ ' bounded by the plane $z=0$ and $z=d$ in a porous medium of porosity $\varepsilon$ and medium permeability $k_{l}$, which is acted upon by the uniform rotation $\Omega(0,0, \Omega)$, uniform vertical magnetic field $H(0,0, H)$ and gravity force $g(0,0,-g)$. The layer is heated from below such that a uniform temperature gradient $\beta\left(=\left|\frac{d T}{d z}\right|\right)$ is maintained.

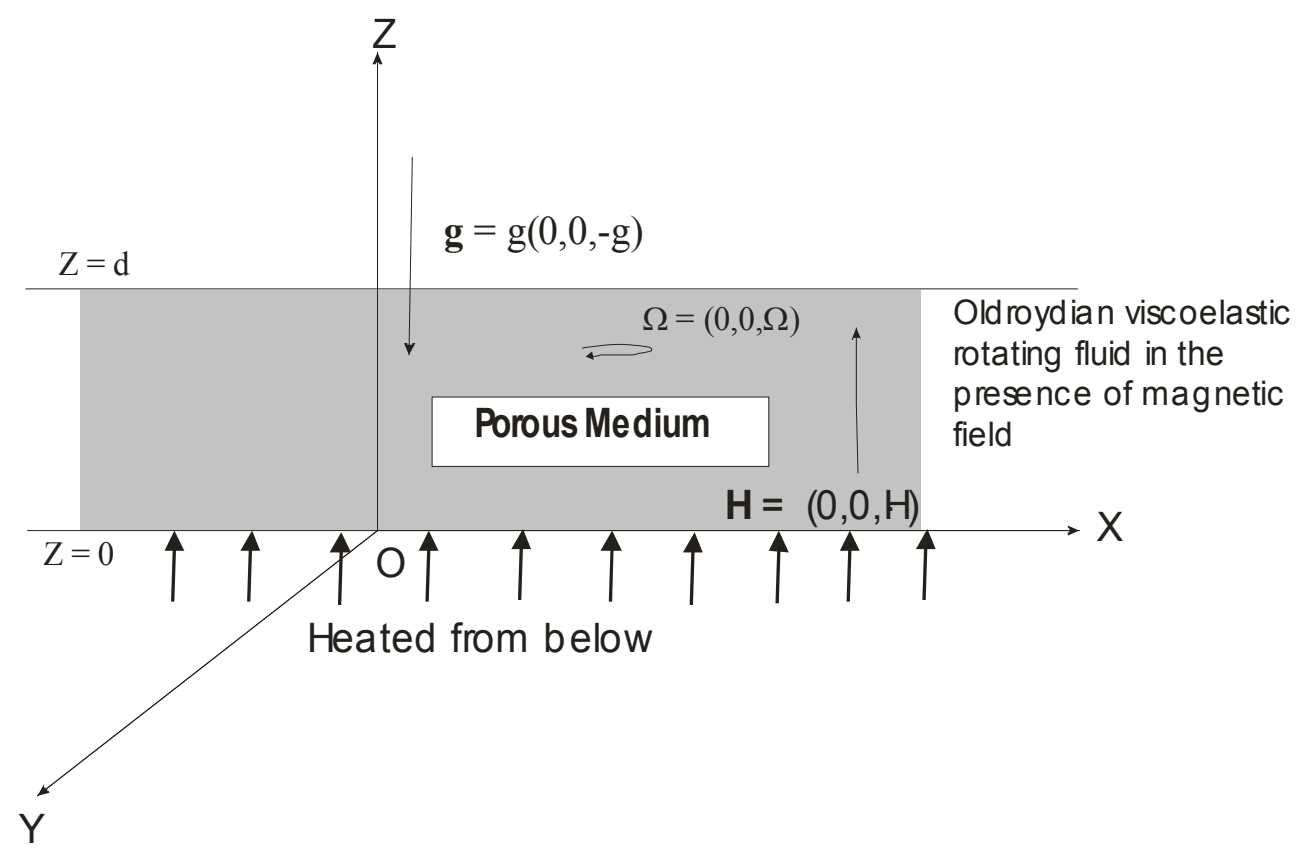

Fig. Schematic sketch of physical situation.

Let $p, \rho, T, \alpha, \mu, \mu_{e}, v, \kappa$ and $\eta$ be the pressure, density, temperature, thermal coefficient of expansion, viscosity, magnetic permeability, kinematic viscosity, thermal diffusivity and resistivity of fluid, respectively.

The equations of motion, continuity and heat conduction for the Oldroydian viscoelastic fluid through a porous medium are

$$
\begin{aligned}
& \frac{\rho}{\varepsilon}\left(1+\lambda \frac{\partial}{\partial t}\right) \frac{d \boldsymbol{q}}{d t}=\left(1+\lambda \frac{\partial}{\partial t}\right)\left[-\nabla p+\rho \boldsymbol{g}+\frac{\mu_{e}}{4 \pi}\{(\nabla \times \boldsymbol{h}) \times \boldsymbol{H}\}+\frac{2 \rho}{\varepsilon}(\boldsymbol{q} \times \boldsymbol{\Omega})\right]+ \\
& -\left(1+\lambda_{0} \frac{\partial}{\partial t}\right)\left(\frac{\mu}{\varepsilon} \nabla^{2}-\frac{\mu}{k_{1}}\right) \boldsymbol{q},
\end{aligned}
$$




$$
\begin{aligned}
& \nabla \cdot \boldsymbol{q}=0 \\
& E \frac{\partial T}{\partial t}+(\boldsymbol{q} \cdot \nabla) T=\kappa \nabla^{2} T .
\end{aligned}
$$

The equation of state is

$$
\rho=\rho_{0}\left[1-\alpha\left(T-T_{0}\right)\right]
$$

where the suffix zero refers to values at the reference level $z=0$, i.e., $\rho_{0}, T_{0}$ stands for density, temperature at lower boundary $z=0$.

Maxwell's equations yield

$$
\varepsilon \frac{d H}{d t}+(\boldsymbol{H} \cdot \nabla) \boldsymbol{q}+\varepsilon \eta \nabla^{2} H
$$

and

$$
\nabla \cdot \boldsymbol{H}=0
$$

where $\frac{d}{d t}=\frac{\partial}{\partial t}+\frac{1}{\varepsilon}(\boldsymbol{q} \nabla)$ stands for the convection derivative.

The steady state solution is

$$
\boldsymbol{q}=(0,0,0), \quad T=T_{0}-\beta z, \quad \rho=\rho_{0}(1+\alpha \beta z)
$$

where $\beta=\left(\frac{T_{0}-T_{1}}{d}\right)$ is the magnitude of uniform temperature gradient, which is maintained.

Let $\delta \rho, \delta p, \theta, \boldsymbol{h}\left(h_{x}, h_{y}, h_{z}\right)$ and $q(u, v, w)$ be the perturbation in density, pressure, temperature, magnetic field and fluid velocity (initially zero), respectively. Then the linearized perturbation equations of flow through a porous medium, following the Boussineq approximations are

$$
\begin{aligned}
& \frac{1}{\varepsilon}\left(1+\lambda \frac{\partial}{\partial t}\right) \frac{\partial \boldsymbol{q}}{\partial t}=\left(1+\lambda \frac{\partial}{\partial t}\right)\left[-\frac{1}{\rho_{0}} \nabla \delta p+g \frac{\delta \rho}{\rho_{0}}+\frac{\mu_{e}}{4 \pi \rho_{0}}\{(\nabla \times \boldsymbol{h}) \times \boldsymbol{H}\}+\frac{2}{\varepsilon}(\boldsymbol{q} \times \Omega)\right]-\frac{v}{\varepsilon}\left(1+\lambda_{0} \frac{\partial}{\partial t}\right)\left(\nabla^{2}-\frac{\varepsilon}{k_{l}}\right) \boldsymbol{q},(2.7) \\
& \nabla \cdot \boldsymbol{q}=0 \\
& \varepsilon \frac{d h}{d t}=(\boldsymbol{H} \cdot \nabla) \boldsymbol{q}+\varepsilon \eta \nabla^{2} h \\
& \nabla \cdot \boldsymbol{h}=0 \\
& E \frac{\partial \theta}{\partial t}=\beta w+\kappa \nabla^{2} \theta
\end{aligned}
$$


The change in density $\delta \rho$ caused by the perturbation in temperature $\theta$ is given by

$$
\delta \rho=-\rho_{0} \alpha \theta \text {. }
$$

In the Cartesian form Eqs (2.7) - (2.11) can be written as

$$
\begin{aligned}
& \frac{1}{\varepsilon}\left(1+\lambda \frac{\partial}{\partial t}\right) \frac{\partial u}{\partial t}=\left(1+\lambda \frac{\partial}{\partial t}\right)\left[-\frac{1}{\rho_{0}} \frac{\partial}{\partial x}(\delta p)+\frac{\mu_{e} H}{4 \pi \rho_{0}}\left\{\frac{\partial h_{x}}{\partial z}-\frac{\partial h_{z}}{\partial x}\right\}+\frac{2}{\varepsilon} \Omega v\right]+ \\
& -\frac{v}{\varepsilon}\left(1+\lambda_{0} \frac{\partial}{\partial t}\right)\left(\nabla^{2}-\frac{\varepsilon}{k_{l}}\right) u \\
& \frac{1}{\varepsilon}\left(1+\lambda \frac{\partial}{\partial t}\right) \frac{\partial v}{\partial t}=\left(1+\lambda \frac{\partial}{\partial t}\right)\left[-\frac{1}{\rho_{0}} \frac{\partial}{\partial y}(\delta p)+\frac{\mu_{e} H}{4 \pi \rho_{0}}\left\{\frac{\partial h_{y}}{\partial z}-\frac{\partial h_{z}}{\partial y}\right\}-\frac{2}{\varepsilon} \Omega u\right]+ \\
& -\frac{v}{\varepsilon}\left(1+\lambda_{0} \frac{\partial}{\partial t}\right)\left(\nabla^{2}-\frac{\varepsilon}{k_{1}}\right) v \\
& \frac{1}{\varepsilon}\left(1+\lambda \frac{\partial}{\partial t}\right) \frac{\partial w}{\partial t}=\left(1+\lambda \frac{\partial}{\partial t}\right)\left[-\frac{1}{\rho_{0}} \frac{\partial}{\partial z}(\delta p)+g \beta \theta\right]-\frac{v}{\varepsilon}\left(1+\lambda_{0} \frac{\partial}{\partial t}\right)\left(\nabla^{2}-\frac{\varepsilon}{k_{l}}\right) w, \\
& \frac{\partial u}{\partial x}+\frac{\partial v}{\partial y}+\frac{\partial w}{\partial z}=0 \\
& \varepsilon \frac{d h_{x}}{d t}=H \frac{\partial u}{\partial z}+\varepsilon \eta \nabla^{2} h_{x}, \\
& \varepsilon \frac{d h_{y}}{d t}=H \frac{\partial v}{\partial z}+\varepsilon \eta \nabla^{2} h_{y}, \\
& \varepsilon \frac{d h_{z}}{d t}=H \frac{\partial w}{\partial z}+\varepsilon \eta \nabla^{2} h_{z}, \\
& \frac{\partial h_{x}}{\partial x}+\frac{\partial h_{y}}{\partial y}+\frac{\partial h_{z}}{\partial z}=0 \\
& E \frac{\partial \gamma}{\partial t}=\beta w+\kappa \nabla^{2} \theta, \\
& \nabla^{2}=\frac{\partial^{2}}{\partial x^{2}}+\frac{\partial^{2}}{\partial y^{2}}+\frac{\partial^{2}}{\partial z^{2}} .
\end{aligned}
$$

Operating Eq.(2.13) by $\frac{\partial}{\partial x}$ and Eq.(2.12) by $\frac{\partial}{\partial y}$; then adding and making use of Eq.(2.16), we get

$$
\frac{1}{\varepsilon}\left(1+\lambda \frac{\partial}{\partial t}\right) \frac{\partial w}{\partial z}=\left(1+\lambda \frac{\partial}{\partial t}\right)\left[\frac{1}{\rho_{0}}\left(\nabla^{2}-\frac{\partial^{2}}{\partial z^{2}}\right)(\delta p)+\frac{\mu_{e} H}{4 \pi \rho_{0}}\left\{\nabla^{2} h_{z}\right\}-\frac{2}{\varepsilon} \Omega s\right]-\frac{v}{\varepsilon}\left(1+\lambda_{0} \frac{\partial}{\partial t}\right)\left(\nabla^{2}-\frac{\varepsilon}{k_{l}}\right) \frac{\partial w}{\partial z} .
$$


Now eliminating $\delta p$ from Eqs (2.15) and (2.22), we get

$$
\frac{1}{\varepsilon}\left(1+\lambda \frac{\partial}{\partial t}\right) \nabla^{2} w=\left(1+\lambda \frac{\partial}{\partial t}\right)\left[g\left(\frac{\partial^{2}}{\partial x^{2}}+\frac{\partial^{2}}{\partial y^{2}}\right) \alpha \theta+\frac{\mu_{e} H}{4 \pi \rho_{0}} \frac{\partial}{\partial z}\left\{\nabla^{2} h_{z}\right\}-\frac{2}{\varepsilon} \Omega \frac{\partial}{\partial z} \varsigma\right]-\frac{v}{\varepsilon}\left(1+\lambda{ }_{0} \frac{\partial}{\partial t}\right)\left(\nabla^{2}-\frac{\varepsilon}{k_{l}}\right) \nabla^{2} w
$$

Again operating Eq.(2.13) by $-\frac{\partial}{\partial y}$ and Eq.(2.12) by $\frac{\partial}{\partial x}$ and adding, we get

$$
\frac{1}{\varepsilon}\left(1+\lambda \frac{\partial}{\partial t}\right) \varsigma=\left(1+\lambda \frac{\partial}{\partial t}\right)\left[\frac{\mu_{e} H}{4 \pi \rho_{0}} \frac{\partial \xi}{\partial z}+\frac{2 \Omega}{\varepsilon} \frac{\partial w}{\partial z}\right]-\frac{v}{\varepsilon}\left(1+\lambda_{0} \frac{\partial}{\partial t}\right)\left(\nabla^{2}-\frac{\varepsilon}{k_{l}}\right) \varsigma .
$$

where $\varsigma=\frac{\partial v}{\partial x}-\frac{\partial u}{\partial y}$, is $z$-component of vorticity and $\xi=\frac{\partial h_{y}}{\partial x}-\frac{\partial h_{x}}{\partial y}$ is $z$-component of current density.

Operating Eq.(2.17) by $-\frac{\partial}{\partial y}$ and Eq.(2.18) by $\frac{\partial}{\partial x}$ and adding, we get

$$
\varepsilon \frac{\partial \varsigma}{\partial t}=H \frac{\partial \zeta}{\partial z} \varepsilon \eta \nabla^{2} \xi
$$

\section{Dispersion relation} the form

Analyzing the disturbances into the normal modes and assuming that the perturbed quantities are of

$$
\left[w, \theta, h_{z}, \varsigma, \xi\right]=[W(z), \Theta(z), K(z), Z(z), X(z)] \exp \left(i k_{x} x+i k_{y} y+n t\right)
$$

where $k_{x}, k_{y}$ are horizontal wave numbers in the $x$ and $y$ directions, respectively, $k^{2}=k_{x}^{2}+k_{y}^{2}$ is the resultant wave number, and $n$ is the growth rate of disturbances.

Using Eq.(3.1), Eqs (2.22) - (2.24), (2.19) and (2.21) become

$$
\begin{aligned}
& \frac{1}{\varepsilon}(1+\lambda n)\left(\frac{d^{2}}{d z^{2}}-k^{2}\right) W=(1+\lambda n)\left[-g k^{2} \alpha \theta+\frac{\mu_{e} H}{4 \pi \rho_{0}}\left(\frac{d^{2}}{d z^{2}}-k^{2}\right) K-\frac{2 \Omega}{\varepsilon} \frac{d Z}{d z}\right]-\frac{v}{\varepsilon}\left(1+\lambda \eta^{n}\right)\left(\frac{d^{2}}{d z^{2}}-k^{2}-\frac{\varepsilon}{k_{l}}\right) W, \\
& \frac{1}{\varepsilon}(1+\lambda n) Z=(1+\lambda n)\left[\frac{\mu_{e} H}{4 \pi \rho_{0}} \frac{d X}{d z}+\frac{2 \Omega}{\varepsilon} \frac{d W}{d z}\right]-\frac{v}{\varepsilon}\left(1+\lambda_{0} n\right)\left(\frac{d^{2}}{d z^{2}}-k^{2}-\frac{\varepsilon}{k_{l}}\right) Z, \\
& \varepsilon n X=H \frac{d Z}{d z}+\varepsilon \eta\left(\frac{d^{2}}{d z^{2}}-k^{2}\right) X, \\
& \varepsilon n K=H \frac{d W}{d z}+\varepsilon \eta\left(\frac{d^{2}}{d z^{2}}-k^{2}\right) K,
\end{aligned}
$$




$$
E n \Theta=\beta W+\kappa\left(\frac{d^{2}}{d z^{2}}-k^{2}\right) \Theta
$$

Equations (3.2) - (3.6) in a non-dimensional form can be written as

$$
\begin{aligned}
& (1+F \sigma)\left[\sigma\left(D^{2}-a^{2}\right) W+\frac{g a^{2} \varepsilon \alpha d^{2}}{v} \Theta \frac{\mu_{e} H d^{3}}{4 \pi \rho_{0} \mathrm{v}}\left(D^{2}-a^{2}\right) D K+\frac{2 \Omega d^{3}}{\mathrm{v}} D Z\right]= \\
& =\left(1+F^{*} \sigma\right)\left(D^{2}-a^{2}-\frac{\varepsilon}{P_{l}}\right)\left(D^{2}-a^{2}\right) W, \\
& \sigma(1+F \sigma)-\sigma\left(1+F^{*} \sigma\right)\left(D^{2}-a^{2}-\frac{\varepsilon}{P_{l}}\right) Z=\left(1+F^{*} \sigma\right) \frac{\mu_{e} \varepsilon H}{4 \pi \rho_{0} \mathrm{v}} D X+(1+F \sigma) \frac{2 \Omega d}{\mathrm{v}} D W, \\
& {\left[D^{2}-a^{2}-\sigma p_{2}\right] K=-\left(\frac{H d}{\varepsilon \eta}\right) D W,} \\
& {\left[D^{2}-a^{2}-\sigma p_{2}\right] X=-\left(\frac{H d}{\varepsilon \eta}\right) D Z,} \\
& {\left[D^{2}-a^{2}-\sigma E p_{1}\right] \Theta=-\left(\frac{\beta d^{2}}{\kappa}\right) W .}
\end{aligned}
$$

Here we have introduced a new coordinate $(x, y, z)=\left(x * d, y^{*} d, z^{*} d\right), D^{*}=d / d z^{*}$ in a new unit of length $d$, thereafter dropping the superscript for simplicity and also putting $a=k d, \sigma=\frac{n d^{2}}{v}, p_{1}=\frac{\nu}{\kappa}$ (Prandtl number) $p_{2}=\frac{v}{\eta}$ (magnetic Prandtl number), $P_{l}=\frac{k_{1}}{d^{2}}$ (dimensionless medium permeability), $F=\frac{\lambda v}{d^{2}}$ (relaxation time parameter) and $F^{*}=\frac{\lambda_{0} v}{d^{2}}$ (retardation time parameter).

Eliminating $K, X, \Theta$ and $Z$ from Eqs (3.7) - (3.11), we get

$$
\begin{aligned}
& \left(D^{2}-a^{2}-\sigma F p_{1}\right)\left(D^{2}-a^{2}\right)\left[\sigma(1+F \sigma)\left(D^{2}-a^{2}-\sigma p_{2}\right)+(1+F \sigma) Q D^{2}-\left(1+F^{*} \sigma\right)\left(D^{2}-a^{2}-\frac{\varepsilon}{P_{l}}\right)\right] \\
& \left\langle\left(D^{2}-a^{2}-\sigma p_{2}\right)\left[\sigma(1+F \sigma)-\left(1+F^{*} \sigma\right)\left(D^{2}-a^{2}-\frac{\varepsilon}{P_{l}}\right)\right]+(1+F \sigma) Q D^{2}\right) W+ \\
& +T(1+F \sigma)\left(D^{2}-a^{2}-\sigma p_{2}\right)^{2}\left(D^{2}-a^{2}-\sigma F p_{1}\right) D^{2} W= \\
& =a^{2} R(1+F \sigma)\left(D^{2}-a^{2}-\sigma p_{2}\right)\left(\left(D^{2}-a^{2}-\sigma p_{2}\right)\left[\sigma(1+F \sigma)-\left(1+F^{*} \sigma\right)\left(D^{2}-a^{2}-\frac{\varepsilon}{P}\right)\right]+(1+F \sigma) Q D\right) W
\end{aligned}
$$


where $\mathrm{R}=\frac{g_{0} \alpha \beta d^{4}}{\kappa \nu}$ is the thermal Rayleigh number, $\mathrm{Q}=\frac{\mu_{e} H^{2} d^{2} \varepsilon}{4 \pi \rho_{0} v \eta}$ is the modified Chandrasekhar number for a porous medium and $\mathrm{T}=\frac{2 \Omega d^{2}}{v}$, is the Taylor number.

Here, we consider the case where both the boundaries are free and perfect conductors of heat, while the adjoining medium is assumed to be electrically non-conducting. Thus the boundary conditions for this case are

$$
W=D^{2} W=D Z=\Theta=0 \quad \text { at } \quad z=0 \quad \text { and } \quad z=1 .
$$

Using the boundary conditions (2.25) it can be shown that all the even order derivatives of $W$ vanish at the boundary and hence the proper solution of Eq.(3.12) characterizing the lowest mode is

$$
W=W_{0} \sin \pi z
$$

where $W_{0}$ is constant.

Substituting (3.14) in Eq.(3.12) and letting

$$
a^{2}=\pi^{2} x, \quad R_{1}=\frac{\mathrm{R}}{\pi^{4}}, \quad i \sigma=\frac{\sigma}{\pi^{2}}, \quad P=\pi^{2} P_{l}, \quad Q_{1}=\frac{\mathrm{Q}}{\pi^{2}}, \quad T_{1}=\frac{\mathrm{T}}{\pi^{4}},
$$

we obtain the following dispersion relation

$$
R_{l}=\frac{1}{x}\left\{\begin{array}{c}
\frac{\left(1+x+i \sigma_{l} E p_{l}\right)(1+x)\left\{\begin{array}{l}
\left(i \sigma_{l}\left(1+i \sigma_{l} \pi^{2} F\right)\left(1+x+i \sigma_{l} p_{2}\right)+\left(1+i \sigma_{l} \pi^{2} F\right)\right) \\
\left(1+i \sigma_{l} \pi^{2} F^{*}\right)\left(1+x+i \sigma_{l} p_{2}\right)\left(1+x+\frac{\varepsilon}{P}\right)
\end{array}\right\}}{\left(1+i \sigma_{l} \pi^{2} F\right)\left(1+x+i \sigma_{l} p_{2}\right)}+ \\
+\frac{T_{l}\left(1+x+i \sigma_{l} p_{2}\right)\left(1+x+i \sigma_{l} E p_{l}\right)}{\left(1+x+i \sigma_{l} p_{2}\right)\left[\left(i \sigma_{l}\left(1+i \sigma_{l} \pi^{2} F\right)+\left(1+i \sigma_{l} \pi^{2} F^{*}\right)\right)\left(1+x+\frac{\varepsilon}{P}\right)\right]+Q_{l}\left(1+i \sigma_{l} \pi^{2} F\right)}
\end{array}\right\} .
$$

\section{The stationary convection}

When the instability sets in as a stationary convection, the marginal state will be characterized by $\sigma=0$. On putting $\sigma=0\left(\sigma_{l}=0\right)$ in Eq.(4.1), it reduces to

$$
R_{1}=\frac{(1+x)}{x}\left[\left(1+x+\frac{\varepsilon}{P}\right)+Q_{1}+\frac{T_{1}(1+x)}{\left(1+x+\frac{\varepsilon}{P}\right)+Q_{1}}\right]
$$

Thus in the stationary convection the stress relaxation parameter $F$ and the strain retardation parameter $F^{*}$ vanish with $\sigma$ and thus the Oldroydian visco-elastic fluid behaves like an ordinary Newtonian fluid.

To study the effect of rotation, the magnetic field and medium permeability, we examine the nature of $\frac{d R_{1}}{d T_{1}}, \frac{d R_{1}}{d Q_{1}}$ and $\frac{d R_{1}}{d P}$ analytically. 
Equation (4.1) yield

$$
\frac{d R_{1}}{d T_{1}}=\frac{(1+x)}{x\left(1+x+\frac{\varepsilon}{P}+Q_{1}\right)}>0 .
$$

Thus rotation has a stabilizing effect on the system in a porous medium. This result is an agreement with the earlier result of Sharma (1975), Rana and Kumar (2010), Rana and Kango (2011).

From Eq.(4.1), we have

$$
\frac{d R_{1}}{d Q_{1}}=\frac{(1+x)}{x}\left[(1+x)\left(1+x+\frac{\varepsilon}{P}+\right)-\frac{T_{I}}{\left(1+x+\frac{\varepsilon}{P}+Q_{1}\right)^{2}}\right]
$$

Hence the magnetic field has a stabilizing if

$$
\left(1+x+\frac{\varepsilon}{P}+\right)>\frac{T_{1}}{\left(1+x+\frac{\varepsilon}{P}+Q_{1}\right)^{2}}
$$

and a destabilizing effect if

$$
\left(1+x+\frac{\varepsilon}{P}+\right)<\frac{T_{1}}{\left(1+x+\frac{\varepsilon}{P}+Q_{1}\right)^{2}} .
$$

This result is an agreement with the earlier result of Sharma (1975). However, in the absence of rotation, the magnetic field completely stabilizes the system.

Also from Eq.(4.1), we get

$$
\frac{d R_{1}}{d P}=-\frac{(1+x)}{x} \frac{\varepsilon}{P^{2}}\left[(1+x) Q_{1}-\frac{T_{I}}{\left(1+x+\frac{\varepsilon}{P}+Q_{I}\right)^{2}}\right] .
$$

Thus, the medium permeability has a stabilizing effect, if

$$
(1+x) Q_{1}<\frac{T_{I}}{\left(1+x+\frac{\varepsilon}{P}+Q_{1}\right)^{2}},
$$

and a destabilizing effect, if

$$
(1+x) Q_{1}>\frac{T_{I}}{\left(1+x+\frac{\varepsilon}{P}+Q_{l}\right)^{2}} .
$$


In the absence of rotation, the medium permeability has a destabilizing effect on the system. This result is an agreement with the earlier results of Sharma (1975), Rana and Kumar (2010), Rana and Kango (2011).

The dispersion relation (4.1) is analyzed numerically. Graphs have been plotted by giving some numerical values to the parameters to depict the stability characteristics.

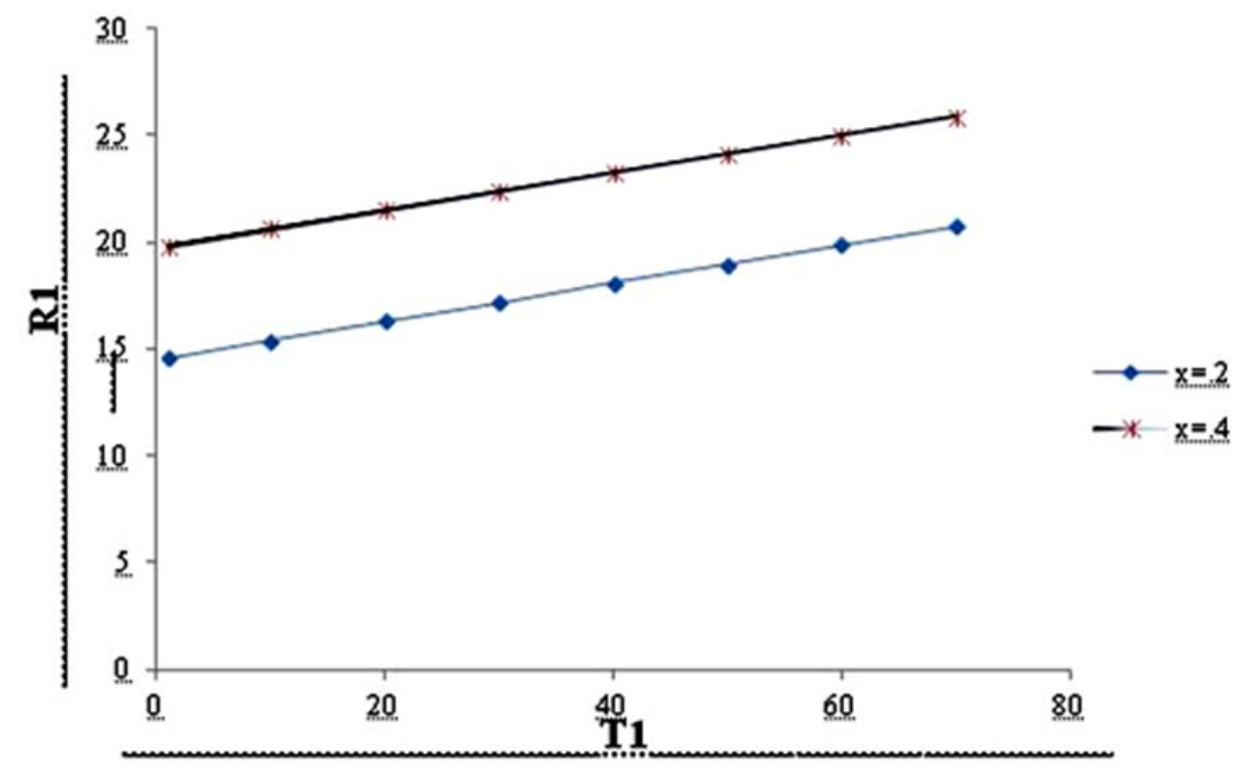

Fig.1. Variation of Rayleigh number $\mathrm{R}_{1}$ with Taylor number $\mathrm{T}_{1}$ for $\varepsilon=0.1, P=10, Q=10$ and for fixed wave numbers $x=0.2$ and $x=0.4$.

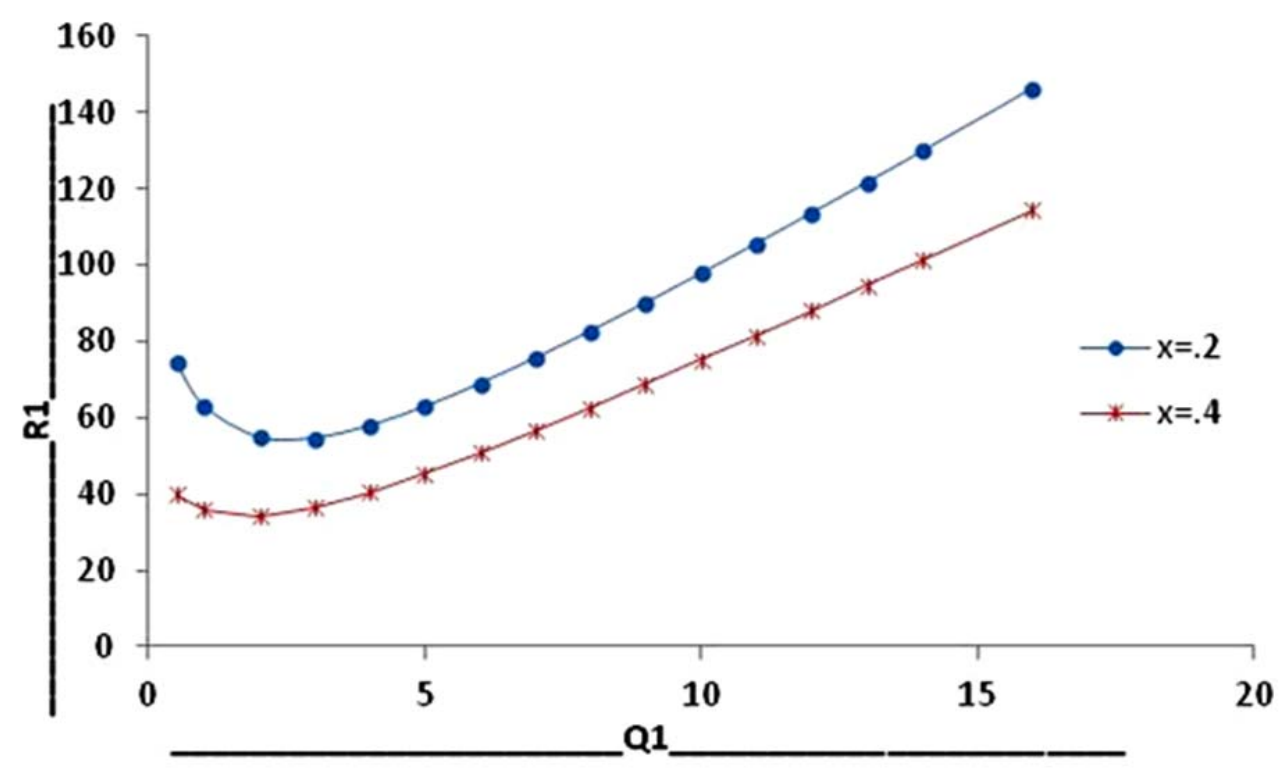

Fig.2. Variation of Rayleigh number $\mathrm{R}_{1}$ with Chandrasekhar number $\mathrm{Q}_{1}$ for $\varepsilon=0.1, P=10, T_{1}=20$ and for fixed wave numbers $x=0.2$ and $x=0.4$. 


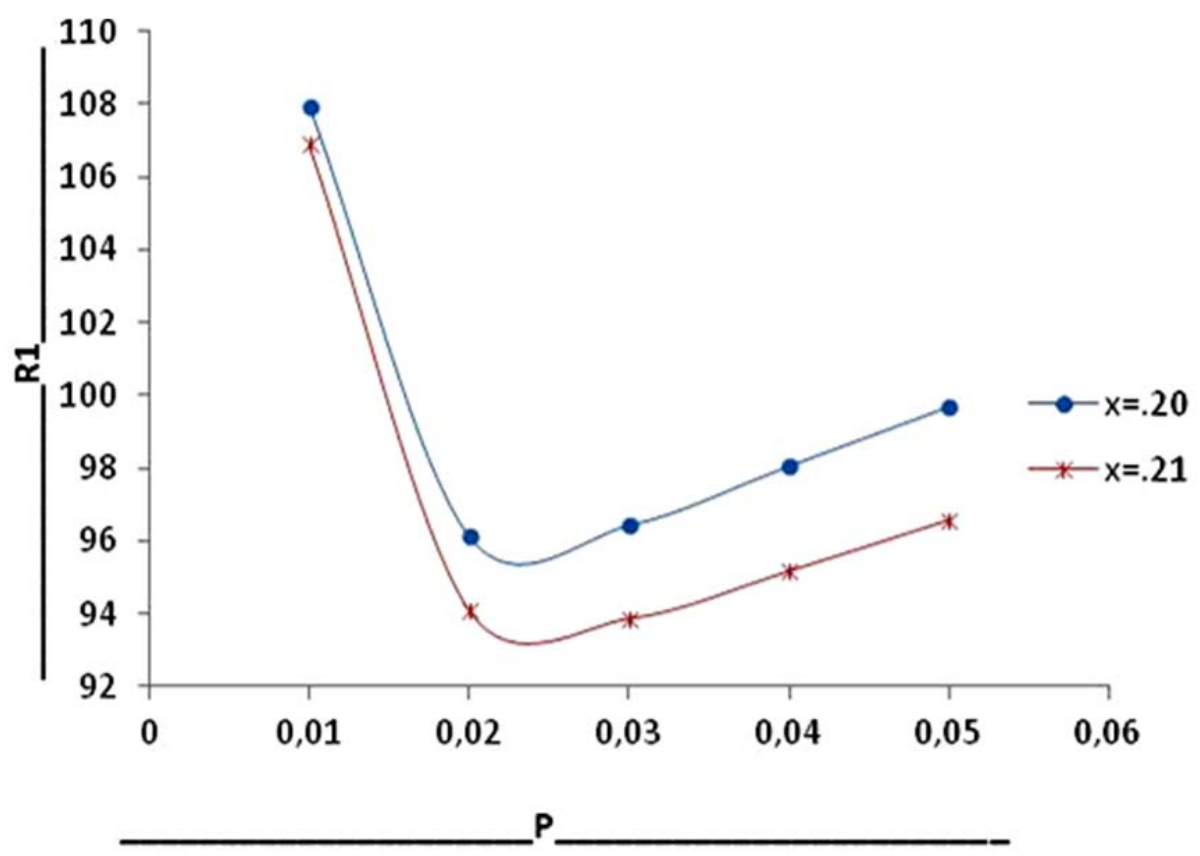

Fig.3. Variation of Rayleigh number $\mathrm{R}_{1}$ with medium permeability $P$ for $\varepsilon=0.1, \mathrm{Q}_{1}=5, \mathrm{~T}_{1}=110$ and for fixed wave numbers $x=0.20$ and $x=0.21$.

In Fig.1, the Rayleigh number $\mathrm{R}_{1}$ is plotted against the Taylor number for $\varepsilon=0.1, P=10, \mathrm{Q}=10$ and for fixed wave numbers $x=0.2$ and $x=0.4$. This shows that $\mathrm{R}_{1}$ increases with the increasing value of $\mathrm{T}_{1}$. Thus, rotation has a stabilizing effect on the system.

In Fig.2, the Rayleigh number $\mathrm{R}_{1}$ is plotted against the Chandrasekhar number $\mathrm{Q}_{1}$ for $\mathrm{Q}_{1}$ for $\varepsilon=0.1, P$ $=10, \mathrm{~T}_{1}=20$ and for fixed wave numbers $x=0.2$ and $x=0.4$. This shows that $\mathrm{R}_{1}$ decreases $/$ increases with the increasing value of $\mathrm{Q}_{1}$, therefore, destabilizing / stabilizing effect of the magnetic field on the system. In Fig.3, The Rayleigh number $\mathrm{R}_{1}$ is plotted against the medium permeability $P$ for $\varepsilon=0.1, \mathrm{Q}_{1}=5, \mathrm{~T}_{1}=110$ and for fixed wave numbers $x=0.20$ and $x=0.21 \mathrm{R}_{1}$ decreases/increases with the increasing value of $P$. Hence, the medium permeability has a destabilizing/stabilizing effect on the system.

\section{Stability of the system and oscillatory modes}

Here, we examine the possibility of oscillatory modes, if any, on the stability problem due to rotation, the magnetic field, gravity field and medium permeability. Multiplying Eq.(3.7) by $W^{*}$ (the complex conjugate of $W$ ), integrating over the range of $z$ and making use of Eqs (3.8) - (3.11) and boundary condition (3.13); we get

$$
\begin{aligned}
& {\left[\sigma(1+F \sigma)+\frac{\varepsilon}{P_{l}}\left(1+F^{*} \sigma\right)\right] I_{1}+\left(1+F^{*} \sigma\right) I_{2}-(1+F \sigma) \frac{g a^{2} d^{2}}{v \beta}\left(I_{3}+\sigma^{*} E_{1} p_{1} I_{4}\right)+} \\
& +\left(1+F^{*} \sigma\right) \frac{\mu_{e} \varepsilon H}{4 \pi \rho_{0} \mathrm{v}}\left(I_{5}+\sigma^{*} p_{2} I_{6}\right)+d^{2}\left[\begin{array}{l}
\sigma^{*}\left(1+F^{*}\right)+\frac{\varepsilon}{P_{l}}\left(1+F^{*} \sigma^{*}\right) I_{7}+ \\
+\left(1+F^{*} \sigma^{*}\right) I_{8}+\frac{\mu_{e} \varepsilon H}{4 \pi \rho_{0} \mathrm{v}}\left(I_{9}+\sigma^{*} p_{2} I_{10}\right)
\end{array}\right] .
\end{aligned}
$$

where 


$$
\begin{aligned}
& I_{1}=\int_{0}^{1}\left(\left.\left|D W^{2}+a^{2}\right| W\right|^{2}\right) d z, \quad I_{2}=\int_{0}^{1}\left(\left|D^{2} W^{t^{2}}+2 a^{2}\right| D W^{2}+a^{4}|W|^{2}\right) d z, \\
& I_{3}=\int_{0}^{1}\left(|D \Theta|^{2}+a^{2}|\Theta|^{2}\right) d z, \quad I_{4}=\int_{0}^{1}\left(|\Theta|^{2}\right) d z, \quad I_{5}=\int_{0}^{1}\left(|D K|^{2}+2 a^{2}|D K|^{2}+a^{4}|K|^{2}\right) d z, \\
& I_{6}=\int_{0}^{1}\left(|D K|^{2}+a^{2}|K|^{2}\right) d z, \quad I_{7}=\int_{0}^{1}\left(|Z|^{2}\right) d z, \quad I_{8}=\int_{0}^{1}\left(|D Z|^{2}+a^{2}|Z|^{2}\right) d z \\
& I_{9}=\int_{0}^{1}\left(|D X|^{2}+a^{2}|X|^{2}\right) d z, \quad I_{10}=\int_{0}^{1}\left(X^{2}\right) d z \text {. }
\end{aligned}
$$

All the integrals from $I_{1}$ to $I_{10}$ are positive definite.

Letting $\sigma=\sigma_{r}+i \sigma_{i}$ in Eq.(5.1), where $\sigma_{r} \sigma_{i}$ is real, and equating the real and imaginary part we have

$$
\begin{aligned}
& \sigma_{r}\left\langle\left[\left(1+F \sigma_{r}\right)+\frac{\varepsilon}{P_{l}} F^{*}\right] I_{1}+F^{*} I_{2} \frac{g a^{2} \alpha \varepsilon d^{2}}{v \beta}\left(I_{3}+E p_{1} I_{4}\right)+\frac{\mu_{e} \varepsilon^{2} \eta}{4 \pi \rho_{0} v}\left(F I_{5}+p_{2} I_{6}\right)+\right. \\
& \left.+d^{2}\left[\left(\left(1+F \sigma_{r}\right)+\frac{\varepsilon}{P_{l}} F^{*}\right) I_{7}+F^{*} I_{8}+\frac{\mu_{e} \varepsilon^{2} \eta}{4 \pi \rho_{0} \mathrm{v}} p_{2} I_{10}\right]\right\rangle= \\
& =-\left\langle\left[\frac{\varepsilon}{P_{l}}-\sigma_{1}^{2} F\right] I_{1} \frac{g a^{2} \alpha \varepsilon d^{2}}{v \beta}\left(I_{3}+\sigma_{r}^{2} E p_{1} I_{4}\right)+\frac{\mu_{e} \varepsilon^{2} \eta}{4 \pi \rho_{0} \mathrm{v}}\left(F I_{5}+p_{2} I_{6}\right)+\right. \\
& \left.+d^{2}\left[\left(\left(1+F \sigma_{r}\right)+\frac{\varepsilon}{P_{l}} F^{*}\right) I_{7}+F^{*} I_{8}+\frac{\mu_{e} \varepsilon^{2} \eta}{4 \pi \rho_{0} v} p_{2} I_{10}\right]\right\rangle .
\end{aligned}
$$

From Eq.(5.3), we conclude that $\sigma_{r}$ is negative or positive which implies that the system may be stable or unstable depending upon certain conditions.

The imaginary part is equal to

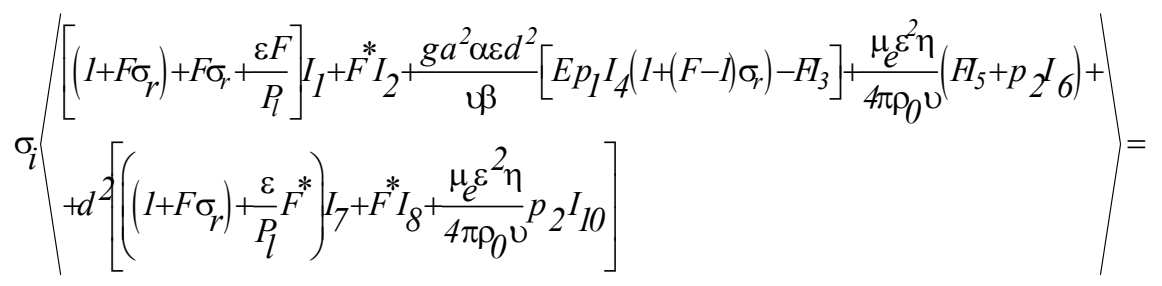

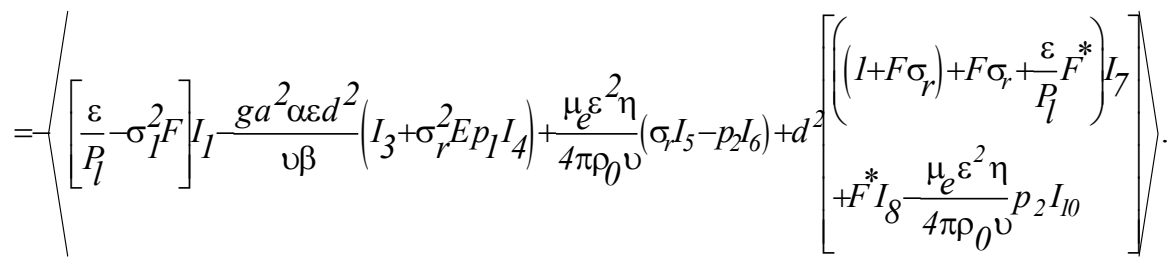

From Eq.(5.4) it is clear that $\sigma i=0$ or $\sigma i \# 0$. Thus modes may be non-oscillatory or oscillatory. 


\section{Principle of exchange of stabilities}

Suppose the principle of exchange of stability is valid, then $\sigma i=0$.

Now put $\sigma i=0$, Eq.(5.4) reduces to

$$
\left[\frac{\varepsilon}{P_{l}}-\sigma_{i}^{2} F\right] I_{1} \frac{g a^{2} \alpha \varepsilon d^{2}}{\mathrm{u} \beta}\left(I_{3}+\sigma_{i}^{2} E p_{1} I_{4}\right)+\frac{\mu_{e} \varepsilon^{2} \eta}{4 \pi \rho_{0} \mathrm{v}}\left(H_{5}+p_{2} I_{6}\right)+d^{2}\left[\left(1+\frac{\varepsilon}{P} F^{*}\right) I_{7}+F^{*} I_{8}+\frac{\mu_{e} \varepsilon^{2} \eta}{4 \pi \rho_{0} \mathrm{v}} p_{2} I_{10}\right]=0 .
$$

Equation (6.1) and Eq.(6.2) are satisfied when $\sigma r=0$. Since $\sigma i=0$ is the necessary condition for the principle of exchange of stabilities Eq.(5.4) is identically satisfied while Eq.(6.1) reduces to

$$
\frac{\varepsilon}{P_{l}} I_{1}-\frac{g a^{2} \alpha \varepsilon d^{2}}{\nu \beta} I_{3}+\frac{\mu_{e} \varepsilon^{2} \eta}{4 \pi \rho_{0} \mathrm{v}}\left(F I_{5}+p_{2} I_{6}\right)+d^{2}\left[\left(1+\frac{\varepsilon}{P_{l}} F^{*}\right) I_{7}+F^{*} I_{8}+\frac{\mu_{e} \varepsilon^{2} \eta}{4 \pi \rho_{0} \mathrm{v}} p_{2} I_{10}\right]=0 .
$$

Again for $\sigma=0$, Eq.(3.11) reduces to

$$
\left(D^{2}-a^{2}\right) \Theta=-\left(\frac{\beta d^{2}}{\kappa}\right) W
$$

Multiplying Eq.(3.11) by $\Theta^{*}$ (complex conjugate of $\Theta$ ), integrating over the range of $z$, applying the boundary conditions (6.3) and separating the real and imaginary parts, we get

$$
\begin{aligned}
& \int_{0}^{1}\left(D^{2}-a^{2}\right) \Theta \Theta^{*} d z=-\left(\frac{\beta d^{2}}{\kappa}\right) \int_{0}^{1} W \Theta^{*} d z \\
& \int_{0}^{1}\left(|D \Theta|^{2}+a^{2}|\Theta|^{2}\right) d z=\operatorname{Re}\left(\frac{\beta d^{2}}{\kappa}\right) \int_{0}^{1} W \Theta^{*} d z, \\
& \operatorname{Re} \int_{0}^{1} W \Theta^{*} d z \leq\left|\int_{0}^{1} \Theta^{*} W d z\right| \\
& \leq \int_{0}^{1}\left|\Theta^{*} W\right| d z, \\
& \leq \int_{0}^{1}\left|\Theta^{*}\right||W| d z, \\
& \leq \sqrt{\left|\Theta^{*}\right|^{2}|W|^{2} d z . \quad \text { (Schwartz inequality) }}
\end{aligned}
$$

Combing inequalities (6.5) and (6.6), we get 


$$
\int_{0}^{1}\left(|D \Theta|^{2}+a^{2}|\Theta|^{2}\right) d z \leq\left(\frac{\beta d^{2}}{\kappa}\right) \sqrt{\left|\Theta^{2}\right|} \sqrt{\left|W^{2}\right| d z}
$$

which implies that

$$
\int_{0}^{1}|D \Theta|^{2} d z \leq\left(\frac{\beta d^{2}}{\kappa}\right) \sqrt{|\Theta|^{2}} \sqrt{|W|^{2}} d z
$$

Since $\int_{0}^{1}|D \Theta|^{2} d z \geq \pi^{2} \int_{0}^{1}|\Theta|^{2} d z$. (Rayleigh Ritz inequality)

Combing inequalities (6.8) and (6.7), we get

$$
\pi^{2} \int_{0}^{l}|\Theta|^{2} d z \leq\left(\frac{\beta d^{2}}{\kappa}\right) \sqrt{|\Theta|^{2}} \sqrt{|W|^{2}} d z
$$

Now combing (6.7) and (6.10), we get

$$
\int_{0}^{1}\left(|D \Theta|^{2}+a^{2}|\Theta|^{2}\right) d z \leq\left(\frac{\beta d^{2}}{\pi \kappa}\right)^{2} \int_{0}^{1}|W|^{2} d z
$$

Since $\quad \int_{0}^{1}\left(|D W|^{2}+a^{2}|W|^{2}\right) d z \geq a^{2} \int_{0}^{1}|W|^{2} d z$.

Using inequalities (6.10)-(6.12), Eq.(3.2) becomes

$$
\begin{aligned}
& \frac{\varepsilon}{P_{l}} I_{1}-\frac{g a^{2} \alpha \varepsilon d^{2}}{v \beta} I_{3}+\frac{\mu_{e} \varepsilon^{2} \eta}{4 \pi \rho_{0} v}\left(F I_{5}+p_{2} I_{6}\right)+d^{2}\left[\left(1+\frac{\varepsilon}{P_{l}} F^{*}\right) I_{7}+F^{*} I_{8}+\frac{\mu_{e} \varepsilon^{2} \eta}{4 \pi \rho_{0} v} p_{2} I_{10}\right]> \\
& a^{2} \varepsilon\left[\frac{1}{P_{l}}-\frac{R}{\pi^{2}}\right]_{0}^{l} \int_{0} \mid W^{2} d z+\frac{\mu_{e} \varepsilon^{2} \eta}{4 \pi \rho_{0} v}\left(F I_{5}+p_{2} I_{6}\right)+d^{2}\left[\left(1+\frac{\varepsilon}{P_{l}} F^{*}\right) I_{7}+F^{*} I_{8}+\frac{\mu_{e} \varepsilon^{2} \eta}{4 \pi \rho_{0} v} p_{2} I_{10}\right]
\end{aligned}
$$

where $R=\frac{g_{0} \alpha \beta d^{4}}{\kappa v}$, is the thermal Rayleigh number.

If $\frac{1}{P_{l}}>\frac{R}{\pi^{2}}$, the RHS inequality (6.13) is positive which implies that LHS of the inequality (6.13) must be positive and therefore Eq.(3.2) cannot be satisfied. Hence, $\frac{1}{P_{l}}>\frac{R}{\pi^{2}}$, is the sufficient condition for the invalidity of the principle of exchange of stabilities. 


\section{Conclusion}

The effect of the magnetic field on the thermal instability of an Oldroydian viscoelastic rotating fluid in a porous medium is investigated. The main conclusions are:

(i) For the case of stationary convection, the Oldroydian viscoelastic fluid behaves like an ordinary Newtonian fluid.

(ii) The expressions for $\frac{d \mathrm{R}_{1}}{d \mathrm{~T}_{1}}, \frac{d \mathrm{R}_{1}}{d \mathrm{Q}_{1}}$ and $\frac{d \mathrm{R}_{1}}{d P}$ are examined analytically and it has been found that rotation has a stabilizing effect while the magnetic field and medium permeability have a stabilizing/destabilizing effect on the system depending upon certain conditions.

(iii) The effect of rotation, the magnetic field and medium permeability on the thermal instability of the Oldroydian fluid in a porous medium has also been shown graphically in Figs 1, 2, and 3.

(iv) The oscillatory modes are introduced due to the presence of rotation, the gravity field and magnetic field which were non-existent in their absence.

(v) In the presence of rotation and the magnetic field, the 'principle of exchange of stabilities' is invalid and the sufficient condition for the invalidity of the 'principle of exchange of stabilities' for the Oldroydian visco-elastic fluid in a porous medium is $\frac{1}{P_{l}}>\frac{R}{\pi^{2}}$.

\section{Nomenclature}

$g$ - gravitational acceleration

$\boldsymbol{g}$ - gravitational acceleration vector

$k$ - wave number of disturbance

$P_{i}-$ dimensionless medium permeability

$p$ - pressure

$p_{I}$ - thermal Prandtl number

$\boldsymbol{q}$ - velocity of fluid

$\alpha-$ thermal coefficient of expansion

$\beta-$ adverse temperature gradient

$\delta-$ perturbation in respective physical quantity

$\varepsilon-$ medium porosity

$\theta-$ perturbation in temperature

$\kappa-$ thermal diffusitivity

$\mu$ - fluid viscosity

$v$ - viscoelasticity

$\varsigma-Z$-component of vorticity

$\boldsymbol{\Omega}$ - rotation vector having components $(0,0, \Omega)$

\section{References}

Bhatia P.K. and Steiner J.M. (1972): Convective instability in a viscoelastic fluid layer. - J. Angew. Math. Mech., vol.52, pp.321-32.

Chandrasekhar S. (1981): Hydrodynamic and Hydromagnetic Stability. - New York: Dover Publications.

Green P. (1968): Convective instability of viscoelastic fluid heated from below. - Phy. Fluids, vol.11, pp.1410.

Ingham D.D. and Pop L. (1981): Transport Phenomena in Porous Media. - New York: Elsevier.

Nield D.A. and Bejan A. (2006): Convection in Porous Medium. - New York: Springer. 
Rana G.C. and Kumar S. (2010): Thermal instability of Rivlin-Ericksen rotating fluid permeated with suspended particles under variable gravity field in porous medium. - Studia Geotechnica et Mechanica, vol. XXXII, pp.3954.

Rana G.C. and Kango S.K. (2011): Effect of rotation on thermal instability of compressible Walters, (Model B') fluid in porous medium. - Vol.3, pp.44-53.

Sharma R.C. (1975): Effect of rotation on thermal instability of a viscoelastic fluid. - Acta Physica Hungarica, vol.40, pp.11-17.

Sharma R.C. (1975): Effect of magnetic field on thermal instability of a viscoelastic fluid. - Acta Physica Hungarica, vol.33, pp.293.

Vafai K. and Hadim H.A. (2000): Hand Book of Porous Media. - New York: Mureel Decker.

Vest C.M. and Arpaci V. (1969): Overstability of viscoelastic fluid heated from below. - J. Fluid Mech., vol.36, pp.613.

Wooding R.A. (1960): Rayleigh instability of a thermal boundary layer in flow through a porous medium. - J. Fluid Mech., vol.9, pp.183-192.

Received: December 2, 2011

Revised: December 12, 2012 